\title{
Erratum: Accuracy of the Quantum Regression Theorem for Photon Emission from a Quantum Dot [Phys. Rev. Lett. 127, 100402 (2021)]
}

\author{
M. Cosacchiø, T. Seidelmann, M. Cygorek, A. Vagov, D. E. Reiter, and V. M. Axt
}

(Received 17 December 2021; published 15 February 2022)

DOI: 10.1103/PhysRevLett.128.079901

In our Letter, the main investigation was a comparison between a numerically exact path-integral (PI) method and the quantum regression theorem (QRT) to model photonic figures of merit in quantum-dot-cavity systems obtained from twotime correlation functions. As a side aspect, we discussed the dependence of applying the quantum regression theorem in different frames, in particular, the lab frame and the polaron frame. The latter was achieved by considering the polaron master equation (PME) approach. Upon closer inspection of our numerical analysis, we have found a parameter discrepancy as explained below that affects the results obtained within the PME approach. We stress that this correction does not affect the majority of the results and the main message of the Letter, namely that the QRT overestimates the phonon influence on the indistinguishability.

Concerning Fig. 2(c) of our original Letter, the numerically exact PI results and the ones obtained by using the QRT in the lab frame were calculated for a quantum dot (QD) with an electron confinement radius of $a_{e}=3 \mathrm{~nm}$ (as defined in Table 1 in Supplemental Material of our Letter [1]). In contrast, the PME results were calculated for $a_{e}=4.175 \mathrm{~nm}$ by mistake, which prohibits a meaningful comparison of the different methods. In Fig. 2(c), the corresponding PME results for a $3 \mathrm{~nm}$ QD are shown (blue dotted line).

Furthermore, in the PME community, two different ways to account for the radiative decay by a phenomenological Lindblad term are being used: either the corresponding rate is scaled by a factor $\langle B\rangle^{2}$ (as done in, e.g., Ref. [85] of our Letter) or not (as done in, e.g., Ref. [87] of our Letter), where $\langle B\rangle$ is the phonon Franck-Condon factor as given in Eq. (15) in Supplemental Material of our Letter. In the calculations shown in our Letter, we had scaled the radiative decay rate by the factor $\langle B\rangle^{2}$. The assumption, though, that the radiative decay of the QD into a spectrally flat electromagnetic field environment is not affected by the phonon environment has recently been confirmed by taking into account both environments microscopically within the numerically exact algorithm ACE [2]. Therefore, we continue here without using the factor $\langle B\rangle^{2}$. This different scaling, though, has only a marginal influence on the corresponding results for our parameters. The different QD size $a_{e}$ is responsible for the larger contribution to the change of the PME results.

As was stated in the original Letter, the QRT applied both in the lab frame and in the polaron frame systematically overestimate the phonon influence on the indistinguishability. But in contrast to the previous comparison, the correct PME results now basically coincide with the QRT results [cf. Fig. 2(c)]. Therefore, applying the QRT in the polaron frame does not improve the accuracy of the indistinguishability.

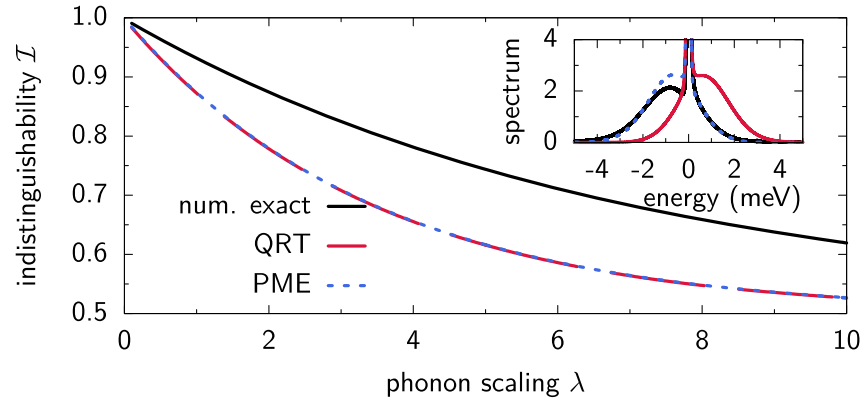

FIG. 2. (c) The indistinguishability as a function of the phonon scaling parameter $\lambda$ at $4 \mathrm{~K}$, calculated with the numerically exact PI method (num. exact), by using the QRT in the lab frame (QRT) and in the polaron frame (PME). Phonon sidebands of the QD emission spectra at $10 \mathrm{~K}$ are shown in the inset. 
To explain this finding, we look at each term of Eq. (4b) of our Letter and compare their derivation within the different methods. The factors in the first term are both (single-time) expectation values of the exciton occupation. We have checked that the PME approach without using the scaling $\langle B\rangle^{2}$ agrees well with the path-integral method regarding the exciton occupation for all parameters concerned. The last term is the second-order two-time correlation function $G^{(2)}(t, \tau)$, upon which the single-photon purity $\mathcal{P}$ is based. In our Letter, we found that the QRT introduces basically no error to this quantity compared with the numerically exact evaluation of the two-time function.

The second term $\left|\left\langle\sigma_{X}^{\dagger}(t+\tau) \sigma_{X}(t)\right\rangle\right|^{2}$ is the absolute square of the first-order correlation function $G^{(1)}(t, \tau)$. Without the factor $\langle B\rangle^{2}$ the PME spectrum is almost a mirror image of the spectrum obtained by applying the QRT in the lab frame [cf. inset of Fig. 2(c)] similar to what was found earlier by a perturbative approach (cf. Ref. [26] of our Letter). This implies that the QRT and PME results differ basically only by the sign of the imaginary part of $G^{(1)}(t, \tau)$, which does not affect the absolute square.

In summary, we find that the QRT and PME agree in most parts and both exhibit deviations by up to $18 \%$ from the numerically exact PI results.

[1] M. Cosacchi, T. Seidelmann, M. Cygorek, A. Vagov, D. E. Reiter, and V. M. Axt, Phys. Rev. Lett. 127, 100402 (2021).

[2] M. Cygorek, M. Cosacchi, A. Vagov, V. M. Axt, B. W. Lovett, J. Keeling, and E. M. Gauger, arXiv:2101.01653. 